Gut and Liver, Vol. 11, No. 4, July 2017, pp. 497-503

\title{
Endoscopic Treatment of Diverse Complications Caused by Laparoscopic Adjustable Gastric Banding: A Study in Eastern Asia
}

Su Young Kim ${ }^{1}$, Kyong Yong $\mathrm{Oh}^{1}$, Jun-Won Chung ${ }^{1}$, Yoon Jae Kim${ }^{1}$, Kyoung Oh Kim¹, Kwang An Kwon ${ }^{1}$, Dong Kyun Park ${ }^{1}$, Kyoung Kon $\mathrm{Kim}^{2}$, and Seong Min $\mathrm{Kim}^{3}$

${ }^{1}$ Divison of Gastroenterology, Department of Internal Medicine, Departments of ${ }^{2}$ Family Medicine and ${ }^{3}$ Surgery, Gachon University Gil Medical Center, Incheon, Korea

Background/Aims: The use of laparoscopic adjustable gastric banding (LAGB) is increasing proportionally with the obesity epidemic. However, some postoperative complications have been highlighted as major problems associated with LAGB. There is no consensus concerning the endoscopic management of these adverse events. The aim of this study was to retrospectively review the feasibility and effectiveness of endoscopic treatment for LAGB complications. Methods: We retrospectively evaluated 352 patients who underwent LAGB between 2011 and 2015. LAGB-associated complications developed in 26 patients (7.4\%). This study involved six patients (1.7\%) who received endoscopic treatment. Results: Types of LAGB-induced complications in our series included intragastric migration $(n=3)$, gastric leaks $(n=2)$, and gastric fistulas $(n=1)$. The endoscopic treatment of these complications was successful in four of the six patients. Endoscopic band removal was successful in two patients. All gastric leaks were successfully closed via an endoscopic procedure. In two cases (intragastric migration and gastric fistula), endoscopic treatment was not sufficient, and surgery was performed. Conclusions: Endoscopic procedures afforded acceptable treatment of band migration and gastric leaks after LAGB. However, the results were poor in patients with gastric fistula. (Gut Liver 2017;11:497-503)

Key Words: Endoscopy; Bariatric surgery; Complication

\section{INTRODUCTION}

Laparoscopic adjustable gastric banding (LAGB) is one of the most popular operations used for the treatment of obesity worldwide. LAGB enables weight loss without gastric resec- tion and, if necessary, provides the opportunity to reinstate the normal anatomy of the stomach. ${ }^{1}$ Despite these advantages, its long-term efficacy has been debated, and several adverse events are associated with band implantation, including band slippage, esophageal dilatation, pouch enlargement, prosthesis infection, and band erosion. ${ }^{2-6}$ These complications, although rare, can be life threatening. Diverse hypotheses have been proposed to interpret these complications, such as an abnormal reaction of the periprosthetic tissue, overfilling of the band, infection, and injury of the gastrointestinal wall during surgery. ${ }^{7-10}$

Upper gastrointestinal (UGI) endoscopy is essential to determine complications associated with LAGB. ${ }^{11}$ The usual treatment for these complications is laparoscopic or open surgery, while endoscopy is rarely used for treatment, but rather diagnostic purposes. Nevertheless, the potential for endoscopic therapy has gradually expanded, especially in cases of band migration. ${ }^{15,6,12,13}$ Because of their minimal invasiveness, safety, and cost-effectiveness, endoscopic procedures are considered a more attractive alternative. Although several studies have demonstrated that endoscopic removal of eroded bands is useful, few have investigated the feasibility of endoscopic treatment for leaks and fistulas caused by LAGB. ${ }^{14,15}$ Moreover, endotherapy of LAGB-induced complications in an East Asian population has not been reported. Thus, we conducted a retrospective study to assess the endoscopic treatment of diverse adverse events in patients who underwent LAGB.

\section{MATERIALS AND METHODS}

A retrospective analysis of all 352 patients who underwent LAGB at the Gil Medical Center between January 2011 and January 2015 was performed to determine those needing en-

Correspondence to: Jun-Won Chung

Division of Gastroenterology, Department of Internal Medicine, Gachon University Gil Medical Center, 21 Namdong-daero 774beon-gil, Namdonggu, Incheon 21565, Korea

Tel: +82-32-460-3778, Fax: +82-32-460-3408, E-mail: junwonchung@hanmail.net

Received on February 20, 2016. Revised on May 7, 2016. Accepted on October 13, 2016. Published online March 24, 2017

pISSN 1976-2283 eISSN 2005-1212 https://doi.org/10.5009/gnl16089

@ This is an Open Access article distributed under the terms of the Creative Commons Attribution Non-Commercial License (http://creativecommons.org/licenses/by-nc/4.0) which permits unrestricted non-commercial use, distribution, and reproduction in any medium, provided the original work is properly cited. 
doscopic treatment. Adverse events developed in 26 of the 352 individuals (7.4\%). Of these, 20 underwent surgery (5.7\%). Of these 20 patients, 12 were diagnosed with minor eroded band problems (less than 20\% of the band lay free in the gastric lumen). It is difficult to access and remove the partially eroded band endoscopically, because the site of erosion is small. In addition, if a partially migrated band is removed endoscopically, there is a high risk of excessive bleeding as the gastric mucosa may be torn further. These 12 patients had small erosion sites, and additionally, some had infections. Therefore, we performed only surgical treatment. Six patients were diagnosed with band slippage, and two had port problems (one infection of the port and one tube disconnection). In general, surgical treatment was indicated for patients with band slippage and tube problems. We performed surgery on eight patients. Therefore, we enrolled only the six remaining patients (1.7\%) who underwent endoscopic management to correct adverse events. An advanced platform standard gastric band had been implanted with laparoscopy for all enrolled patients. All bands were inserted via the pars flaccida route, and the subfascial technique was used for port fixation. The average volume of saline injected into the band was $6 \mathrm{~mL}$.

LAGB-associated complications were confirmed by an UGI series using a water-soluble contrast medium (Gastrografin; Bayer Vital, Leverkusen, Germany), esophagogastroduodenoscopy (EGD), and contrast-enhanced computed tomography of the abdomen and pelvis. Based on a study by Blero et al., the degree of band dysfunction was classified as follows: type I, slipping of the band; type II, stenosis with pouch dilatation; type IIIa, minor erosion; and type IIIb, major erosion or intragastric migration. The endoscopic procedure was performed in the endoscopy unit using an endoscope (GIF-Q260 or GIF-H260; Olympus Medical Systems Corp., Tokyo, Japan). Propofol, given as an intravenous bolus, was used for sedation. Antibiotics were administered in leakage and fistula cases.

The endoscopic treatment was selected based on the type of adverse event, including eroded bands, gastric leaks, and fistulas. In the event of band migration, endoscopic access via the Gastric Band Cutter (Agency for Medical Innovations $\mathrm{GmbH}$,
Feldkirch, Austria) was used to extirpate the migrated band. The endoscopic treatment method for removing an eroded band is described below. We first confirmed that the migrated band entered the stomach and that the migration was over 50\%. The port was removed surgically under local anesthesia, and the cutting wire was introduced into the stomach through the hole of the endoscope and held in the antrum. The endoscope was then reintroduced and passed inside the migrated band to regain the wire, and a loop was formed around the eroded band. A metal sheath was introduced at the end of the wire, which was inserted into the stomach opposite the band. By turning the handgrip of the Gastric Band Cutter, the band was cut and extracted using a net snare with the endoscope. A variety of methods were employed for the treatment of gastric leaks and fistulas, including tissue adhesives, tubes, and stents. The purpose of the treatment was to drain fluid collection sufficiently and to correct the leakage and fistula.

We retrospectively reviewed the patient characteristics, surgical techniques, and methods of endoscopic treatment for each patient in addition to the stage of band erosion, further complications, length of hospital stay, and the time interval between the adverse event and initial LAGB placement. Data were expressed as medians and ranges. The retrospective review of these cases was approved by the Institutional Review Board of the Gil Medical Center (IRB number: GBIRB 2015-265).

\section{RESULTS}

From November 2011 to June 2014, six patients (mean age, 38.2 years [range, 24 to 56 years]; six females) were treated by endoscopy at the Gil Medical Center for band erosion or gastric leaks or fistulas. The clinical characteristics of the study population are shown in Table 1 . The median time between LAGB and the diagnosis of the complication was 26 months (range, 3 to 49 months). The most common symptom was epigastric pain. In one patient, obesity was accompanied by diabetes mellitus. Three patients (50.0\%) underwent bariatric surgery twice.

In three patients (number 1 to 3), removal of the eroded band

Table 1. Patient Characteristics

\begin{tabular}{|c|c|c|c|c|c|c|c|c|}
\hline $\begin{array}{c}\text { Patient } \\
\text { no. }\end{array}$ & Sex & $\begin{array}{l}\text { Age, } \\
\text { yr }\end{array}$ & Comorbidities & Type of bariatric surgery & $\begin{array}{c}\text { Dysfunction } \\
\text { type }\end{array}$ & Adverse events & $\begin{array}{l}\text { Time to adverse } \\
\text { events after the } \\
\text { first LAGB, mo }\end{array}$ & Presenting symptoms \\
\hline 1 & $\mathrm{~F}$ & 32 & - & LAGB & IIIlb & Intragastric migration & 38 & Epigastric pain \\
\hline 2 & $\mathrm{~F}$ & 56 & - & LAGB & IIIb & Intragastric migration & 25 & Epigastric pain, dysphagia \\
\hline 3 & $\mathrm{~F}$ & 43 & - & LAGB & IIIlb & Intragastric migration & 49 & Nausea \\
\hline 4 & $\mathrm{~F}$ & 37 & - & 1st LAGB/ 2nd LAGB & IIIla & Gastric leak & 3 & Epigastric pain \\
\hline 5 & $\mathrm{~F}$ & 37 & DM & 1st LAGB/ 2nd LAGB & IIIla & Gastric fistula & 27 & Abdominal pain, band site infection \\
\hline \multirow[t]{2}{*}{6} & $\mathrm{~F}$ & 24 & - & 1st LAGB/ & I & Gastric leak & 9 & Abdominal pain, nausea, vomiting \\
\hline & & & & 2nd sleeve gastrectomy & & & & \\
\hline
\end{tabular}

LAGB, laparoscopic adjustable gastric banding; F, female; DM, diabetes mellitus. 
using an endoscopic approach was the preferred treatment since the banding tube was discovered entering the cardia of the stomach. In one patient (number 1), the band was successfully severed using the Gastric Band Cutter and extracted using a net snare. In another patient (number 2), we attempted to remove the migrated gastric band by cutting the wire using the Gastric Band Cutter; however, the band did not break completely, and the wire became disconnected. Fortunately, the band buckle had entered the stomach. Thus, the band's proximal tube was cut using endoscopic scissors, and the band buckle was untied using rat-tooth forceps. The band was then carefully removed using a hanging snare without further complications (Fig. 1). In patient number 3, removal of the band using the Gastric Band Cutter, endoscopic scissors, and an electrosurgical device proved impossible, as the band was firmly fixed in place by adhesions, and we were unable to position our removal devices optimally. Thus, surgery was performed to remove the band.

In two patients, slipping of the gastric band occurred after the initial LAGB surgery; thus, they underwent a second LAGB surgery after removal of the initial displaced band. After the second surgery, either a gastric leak or fistula occurred at the posteroinferior site. In one patient (number 4), a gastric leak was found 4 days after the second LAGB surgery. The band was removed immediately by open abdominal surgery, and a T-tube was inserted into the leakage site to create a controlled fistula. After 10 weeks, the T-tube was removed by cutting using endoscopic scissors and retrieved using endoscopic forceps. Fibrin glue (2 mL, Greenplast; Green Cross Corp., Seoul, Korea) was then injected into the leakage site (Fig. 2), and no further leaks were identified. In the other patient (number 5), a gastrocutaneous fistula and a collection of intra-abdominal fluid were found 15 months after the second LAGB surgery. The band was removed promptly, and percutaneous drainage of the fluid was performed. An over-the-scope clip (OTSC) (Ovesco Endoscopy USA Inc., Cary, NC, USA) was used to attempt to close the fistula. However, the UGI contrast series revealed that the leak persisted; thus, an $8 \mathrm{~cm}$ covered self-expandable metal stent was deployed across the fistula by using Shim's technique (Fig. 3). ${ }^{16}$ Thereafter, the leak and fistula remained; thus, the patient was referred for surgery.

Six months after LAGB surgery, band slipping occurred in patient number 6; thus, the band was removed surgically. Two months after, sleeve gastrectomy was performed, and fibrosing and adhesion of the upper body of the stomach were observed
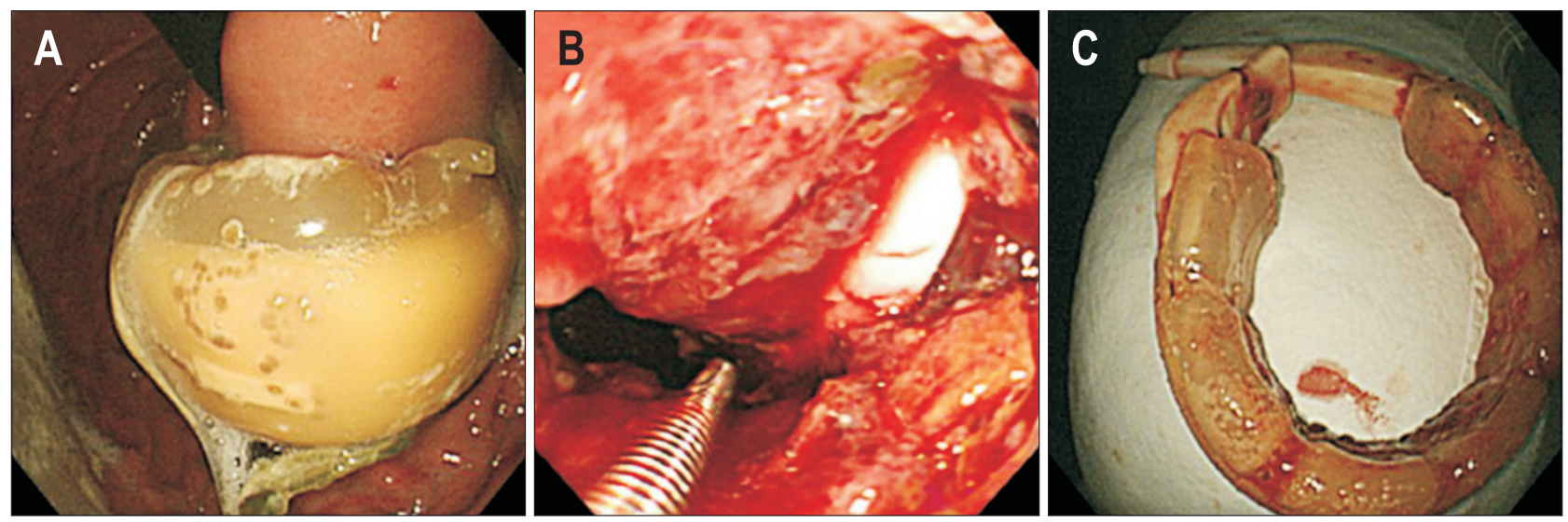

Fig. 1. Endoscopic images from patient number 2. (A) Endoscopic appearance of band migration (dysfunction type IIIb). (B) Endoscopic removal was attempted when the buckle of the band entered the stomach. (C) Endoscopic view of a band removed via the mouth. The band had not been cut and was removed intact.

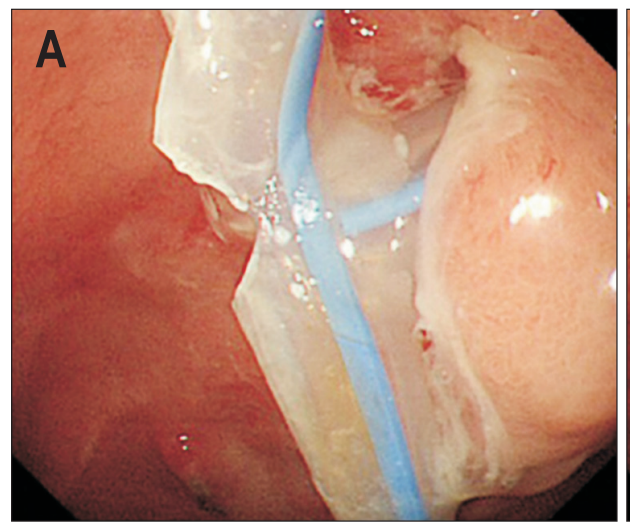

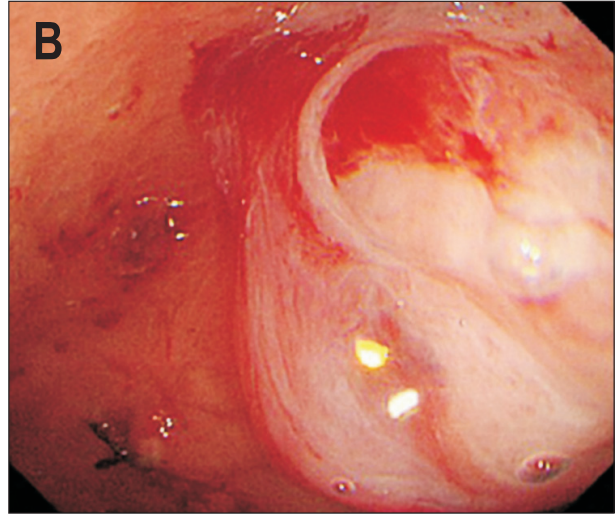

Fig. 2. Endoscopic images from patient number 4. (A) A T-tube was placed in the leakage site. (B) After the tube was removed, fibrin glue was injected into the fistula. 

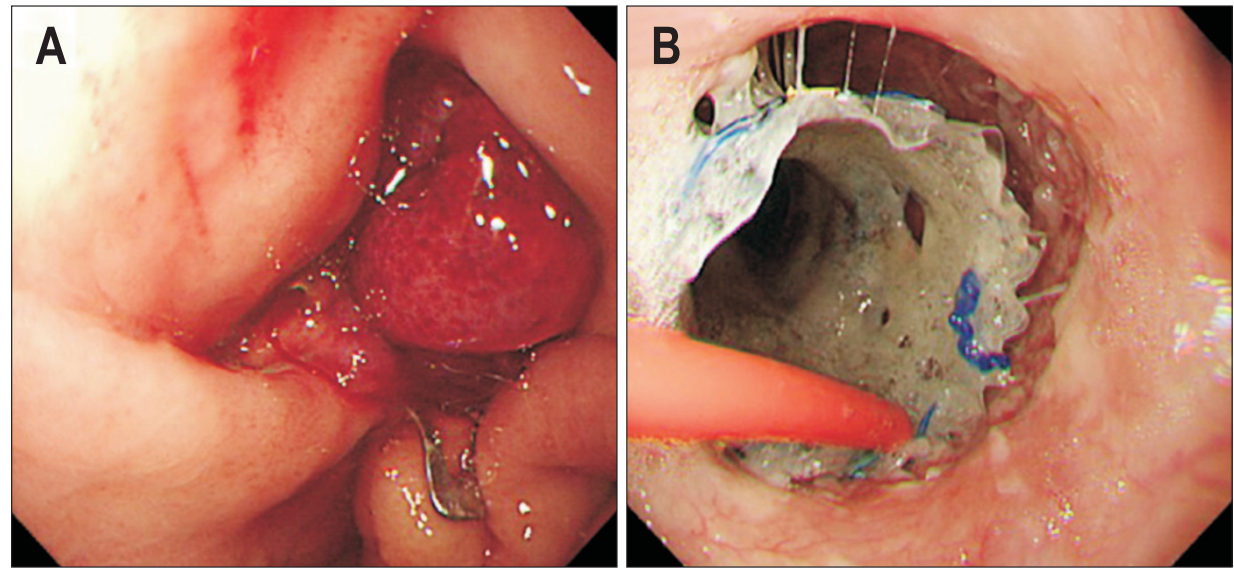

Fig. 3. Images of the fistula after the second bariatric surgery in patient number 5. (A) An over-thescope clip was applied to the fistula. (B) Endoscopic images of the selfexpandable metal stent (Shim's technique) across the fistula.
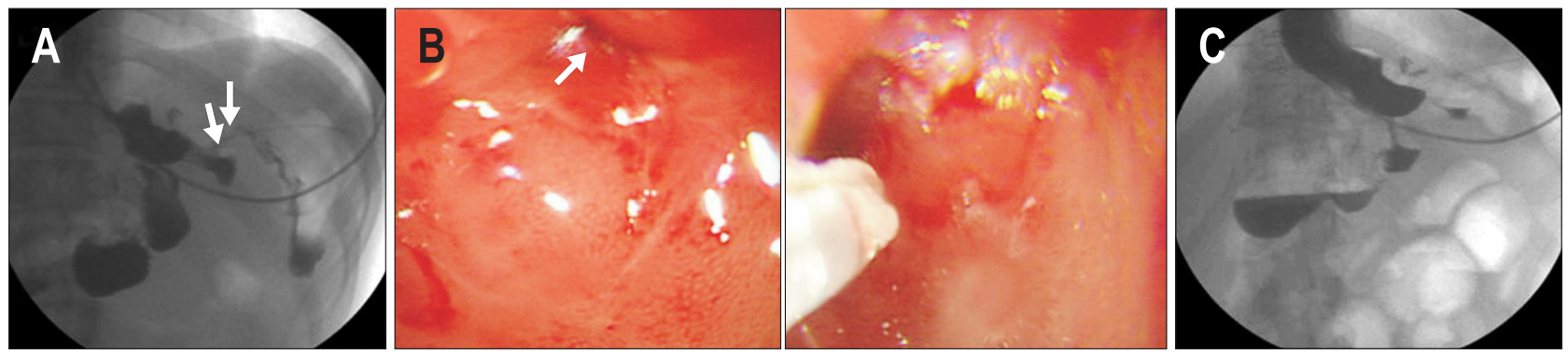

Fig. 4. Images of the leak after the second bariatric surgery in patient number 6. (A) An upper gastrointestinal (UGI) image of the gastric leak. The arrows show the contrast material outside the stomach. (B) Endoscopic view of cyanoacrylate injection into the leakage site. The arrow shows the leak orifice. (C) UGI image showing no visible contrast through the stomach wall.

Table 2. Overview of Patients Who Underwent Endoscopic Treatment

\begin{tabular}{|c|c|c|c|c|c|c|}
\hline Patient no. & $\begin{array}{l}\text { Time from diagnosis } \\
\text { to ET, wk }\end{array}$ & $\begin{array}{c}\text { Total ET time, } \\
\text { min/no. }\end{array}$ & $\begin{array}{c}\text { Endoscopic } \\
\text { success }\end{array}$ & $\begin{array}{c}\text { Further } \\
\text { complications }\end{array}$ & $\begin{array}{l}\text { Meal resumption time } \\
\text { after ET, day }\end{array}$ & LOS, day \\
\hline 1 & $<1$ & $124 / 1$ & Yes & No & 1 & 3 \\
\hline 2 & $<1$ & $72 / 1$ & Yes & No & 1 & 2 \\
\hline 3 & $<1$ & $160 / 1$ & No & No & 3 & 5 \\
\hline 4 & 10 & $35 / 1$ & Yes & No & 1 & 85 \\
\hline 5 & $<1$ & $64 / 2$ & No & No & 23 & 84 \\
\hline 6 & $<1$ & $40 / 1$ & Yes & No & 6 & 32 \\
\hline
\end{tabular}

ET, endoscopic treatment; LOS, length of hospital stay.

where the previous band had been placed. Two weeks after the operation, a gastric leak of the upper body was confirmed by a UGI contrast series and EGD. A 2-mg mixture of cyanoacrylate (Histoacryl; B. Braun Surgical, Rubi, Spain) and lipiodol was administered to inhibit the gastric leak (Fig. 4). The patient recovered completely with conservative therapy and without any additional surgical interventions.

We attempted endoscopic management in six patients, of whom the procedure was successful in four (66.7\%). The median time from diagnosis to endoscopic management was 1 week, but in one case, a period of 10 weeks was required to control the fistula. The median value of the duration of endoscopic treatment was 68 minutes (range, 35 to 160 minutes). The me- dian number of endoscopic procedures was 1 (range, 1 to 2). There were no adverse effects or stent migration associated with the procedures. The median duration to resumption of oral feeding was 2 days (range, 1 to 23 days) after endoscopic treatment, and the median duration of hospitalization was 18.5 days (range, 2 to 85 days) (Table 2). No patients died during the perioperative or follow-up periods.

\section{DISCUSSION}

This is the first retrospective case series, to our knowledge, demonstrating the feasibility of endoscopic treatment for various adverse effects due to LAGB surgery in an East Asian 
population. This study revealed the effects of endoscopic treatment for various complications after LAGB. The success rate was $66.7 \%$, and no complications due to endoscopic treatment were observed. The majority of endoscopic procedures were performed within one session, with short procedure duration. In addition, comparatively fast meal resumption was also possible after the procedure.

LAGB surgery is one of the most common methods of bariatric surgery performed in Western countries. ${ }^{17}$ The advantages of this operation are as follows: the size of the band can be adjusted, the surgical procedure is simple, the associated morbidity and mortality are very low, and previous anatomy may be restored if required by band removal. ${ }^{18}$ However, despite these advantages, reoperation is commonly required due to complications associated with the band. ${ }^{19}$ As a result, LAGB has gradually declined in Europe. ${ }^{17}$ Unlike in Western countries, severely obese individuals who require bariatric surgery are not common in Asia. Thus, few studies have investigated the endoscopic management of LAGB complications in Asians.

Intragastric migration of the band after LAGB is rare, and an incidence of less than $1 \%$ has been reported. ${ }^{20}$ No consensus exists concerning the appropriate management of band erosion, including intragastric migration. Although many surgeons suggest a laparoscopic approach for removal of an eroded band, ${ }^{21}$ recent studies demonstrate a high success rate using an endoscopic approach. ${ }^{1,5,12,13}$ Compared with surgery, an endoscopic approach has several advantages, as it is minimally invasive, prevents unnecessary incision, saves time, results in quick recovery, and is a safe procedure with only minor complications. Some patients may require special conditions for endoscopic band removal. For example, as with patient number 2 in our study, it may be possible to cut the band, as the buckle must be visible. Moreover, it is important to place the endoscopic device ideal for removal of the eroded band. Patient number 3 required surgery, because firm perigastric adhesion prevented endoscopic band removal, and similar cases have been reported in previous studies. ${ }^{1,5}$ Presumably, a long postoperative period (i.e., 4 years in our patient) may result in severe adhesions.

Leakages rarely appear as complications of LAGB. According to a large prospective study, leaking occurred in only one patient $(0.05 \%, 1 / 2,097) .{ }^{22}$ Leaks are classified as acute $(<7$ days), early ( 1 to 6 weeks), late (6 to 12 weeks), or chronic ( $>12$ weeks) $;^{23}$ the stage of both of our patients (number 4 and 6) was acute. In patient number 4, we inserted a T-tube into the leakage site, which permitted control of the leak and resulting in a controlled fistula. We concluded that the T-tube has a similar function to the stent, providing a shield between the impaired site and the acidic enteric contents. It will keep the anastomosis patent may stem wound contraction and stenosis. Moreover, it can improve the nutritional status of patients as it enables early initiation of enteral feeding. ${ }^{24}$ Fibrin glue application is regarded as effective for the management of small leakages and fistulas, ${ }^{25}$ and the use of fibrin glue and cyanoacrylate glue for treatment in patients number 4 and 6, respectively, was successful. Fibrin glue can be applied easily to various locations; however, additional procedures, such as placements of clips or stents, may be required in some cases. In addition, complications of the UGI tract may heal faster than do those of the lower gastrointestinal tract. ${ }^{25}$ In our patient's case, the fibrin glue was applied to the stomach and to the T-tube together, and the leak was treated easily. A review article demonstrated that cyanoacrylate glue has been used successfully in many studies for endoscopic treatment of various leaks and fistulas of the gastrointestinal tract. ${ }^{26,27}$ Cyanoacrylate glue treatment, similar to fibrin glue treatment, is required for stent combination therapy to obtain more effective results. ${ }^{26-28}$ In our study (patient number 6), the application of cyanoacrylate appeared to be efficacious in the treatment of the leakage, although a stent was not used. We detected the leakage early and treated it without delay. In addition, cyanoacrylate is more durable than is fibrin glue, as it is not degraded by pancreatic enzymes or gastric acid. ${ }^{29}$

We were unable to treat the gastrocutaneous fistula of patient number 5, even after various endotherapy attempts. Some studies have demonstrated that the use of the OTSC yields promising results, with a greater than $80 \%$ closure rate for leaks and fistulas. ${ }^{30,31}$ However, the OTSC has several limitations, including the need for several endoscopy sessions and additional endoscopic management (tissue adhesives, stents, etc.), and the fact that it may not apply to larger leaks. ${ }^{32}$ Shim's technique has preventative effects on stent migration, ${ }^{16}$ but its role was indistinctive in our case. We were unable to resolve the fistula for several reasons. First, treatment success rates after stenting are lower for fistulas than for acute leaks. ${ }^{24}$ Second, the fistula was found late and was already irreversible histologically. Third, our patient had a gastrocutaneous fistula. Some studies have reported endotherapy using tissue adhesives for gastrocutaneous fistula that occurred after bariatric surgery. ${ }^{33,34}$ However, another study demonstrated that cure of gastrocutaneous fistulas was more difficult than for other types of fistula. ${ }^{35}$ Therefore, the type of fistula may also have affected the treatment results. Finally, it was difficult to achieve full closure of the fistula using a stent, as the stent diameter was too small to overlay the gastric side of the fistula. Therefore, tissue hyperplasia of the gastric side did not fully develop.

If the first LAGB surgery fails, surgeons may consider replacing the band with a new band or performing another type of bariatric surgery. Although it has been reported that reoperation after LAGB is safe and feasible, we were worried that the history of LAGB may increase the risk of leakage. ${ }^{36}$ The scar tissue and adhesion around the band provoked inadequate healing of the stomach after the second bariatric operation. Moreover, LAGB may exacerbate the vascular supply of the esophagogastric junction, where most leaks arise. ${ }^{37}$ For these reasons, stapling is not always durable, and leaks may occur easily. In the case of 
patient number 6, the leak occurred after sleeve gastrectomy, as LAGB led to severe fibrosis and adhesion. Another study demonstrated that the risk of leakage after conversion to sleeve gastrectomy due to LAGB failure was not increased when conducted as a two-step procedure with satisfactory interval periods. $^{37}$

Our study had certain limitations, including its retrospective nature and the small sample size. In addition, endoscopic treatment failed in some patients, especially those with gastric fistula. Therefore, we currently lack sufficient evidence to claim that endoscopic procedures can be used to treat all types of LAGBinduced complications.

In conclusion, we have shown that endoscopic treatment is an attractive therapeutic option when managing various complications caused by LAGB. It allows for early resumption of oral intake and precludes surgery. Moreover, it is a minimally invasive technique and a simple solution for potentially complex adverse events. Based on our results and other recent studies, we recommend that endoscopic treatment could be considered as the first option for patients exhibiting intragastric band migration or gastric leaks after LAGB. However, our sample size was small, and endotherapy was not effective in all patients. Thus, more data from larger high-quality studies are needed to evaluate the feasibility of endoscopic procedures.

\section{CONFLICTS OF INTEREST}

No potential conflict of interest relevant to this article was reported.

\section{ACKNOWLEDGEMENTS}

This research was supported by a grant of the Korea Health Technology R\&D Project through the Korea Health Industry Development Institute (KHIDI), funded by the Ministry of Health \& Welfare, Republic of Korea (grant number: HI16C2319).

\section{REFERENCES}

1. Mozzi E, Lattuada E, Zappa MA, et al. Treatment of band erosion: feasibility and safety of endoscopic band removal. Surg Endosc 2011;25:3918-3922.

2. Nocca D, Frering V, Gallix B, et al. Migration of adjustable gastric banding from a cohort study of 4236 patients. Surg Endosc 2005;19:947-950.

3. Favretti F, Cadière GB, Segato G, et al. Laparoscopic banding: selection and technique in 830 patients. Obes Surg 2002;12:385390.

4. Mittermair RP, Weiss H, Nehoda H, Kirchmayr W, Aigner F. Laparoscopic Swedish adjustable gastric banding: 6-year follow-up and comparison to other laparoscopic bariatric procedures. Obes Surg 2003;13:412-417.
5. Blero D, Eisendrath P, Vandermeeren A, et al. Endoscopic removal of dysfunctioning bands or rings after restrictive bariatric procedures. Gastrointest Endosc 2010;71:468-474.

6. Di Lorenzo N, Lorenzo M, Furbetta F, et al. Intragastric gastric band migration: erosion: an analysis of multicenter experience on 177 patients. Surg Endosc 2013;27:1151-1157.

7. Abu-Abeid S, Keidar A, Gavert N, Blanc A, Szold A. The clinical spectrum of band erosion following laparoscopic adjustable silicone gastric banding for morbid obesity. Surg Endosc 2003;17:861-863.

8. Niville E, Dams A, Vlasselaers J. Lap-Band erosion: incidence and treatment. Obes Surg 2001;11:744-747.

9. Meir E, Van Baden M. Adjustable silicone gastric banding and band erosion: personal experience and hypotheses. Obes Surg 1999;9:191-193.

10. Biagini J. Intragastric band erosion. Obes Surg 2001;11:100.

11. Lattuada E, Zappa MA, Mozzi E, et al. Band erosion following gastric banding: how to treat it. Obes Surg 2007;17:329-333.

12. Dogan ÜB, Akin MS, Yalaki S, Akova A, Yilmaz C. Endoscopic management of gastric band erosions: a 7-year series of 14 patients. Can J Surg 2014;57:106-111.

13. Chisholm J, Kitan N, Toouli J, Kow L. Gastric band erosion in 63 cases: endoscopic removal and rebanding evaluated. Obes Surg 2011;21:1676-1681.

14. Iacopini F, Di Lorenzo N, Altorio F, Schurr MO, Scozzarro A. Overthe-scope clip closure of two chronic fistulas after gastric band penetration. World J Gastroenterol 2010;16:1665-1669.

15. Qadeer MA, Dumot JA, Vargo JJ, Lopez AR, Rice TW. Endoscopic clips for closing esophageal perforations: case report and pooled analysis. Gastrointest Endosc 2007;66:605-611.

16. Shim CS, Cho YD, Moon JH, et al. Fixation of a modified covered esophageal stent: its clinical usefulness for preventing stent migration. Endoscopy 2001;33:843-848.

17. Buchwald H, Oien DM. Metabolic/bariatric surgery Worldwide 2008. Obes Surg 2009;19:1605-1611.

18. Dixon JB, Dixon ME, O'Brien PE. Pre-operative predictors of weight loss at 1-year after Lap-Band surgery. Obes Surg 2001;11:200-207.

19. Himpens J, Cadière GB, Bazi M, Vouche M, Cadière B, Dapri G. Long-term outcomes of laparoscopic adjustable gastric banding. Arch Surg 2011;146:802-807.

20. Baldinger R, Mluench R, Steffen R, Ricklin TP, Riedtmann HJ, Horber FF. Conservative management of intragastric migration of Swedish adjustable gastric band by endoscopic retrieval. Gastrointest Endosc 2001;53:98-101.

21. Cherian PT, Goussous G, Ashori F, Sigurdsson A. Band erosion after laparoscopic gastric banding: a retrospective analysis of 865 patients over 5 years. Surg Endosc 2010;24:2031-2038.

22. Kohn GP, Hansen CA, Gilhome RW, McHenry RC, Spilias DC, Hensman C. Laparoscopic management of gastric band erosions: a 10-year series of 49 cases. Surg Endosc 2012;26:541-545.

23. Rosenthal RJ; International Sleeve Gastrectomy Expert Panel, Diaz AA, et al. International Sleeve Gastrectomy Expert Panel Con- 
sensus Statement: best practice guidelines based on experience of $>12,000$ cases. Surg Obes Relat Dis 2012;8:8-19.

24. Walsh C, Karmali S. Endoscopic management of bariatric complications: a review and update. World J Gastrointest Endosc 2015;7:518-253.

25. Lippert E, Klebl FH, Schweller F, et al. Fibrin glue in the endoscopic treatment of fistulae and anastomotic leakages of the gastrointestinal tract. Int J Colorectal Dis 2011;26:303-311.

26. ASGE Technology Committee, Bhat YM, Banerjee S, et al. Tissue adhesives: cyanoacrylate glue and fibrin sealant. Gastrointest Endosc 2013;78:209-215.

27. Vakalopoulos KA, Daams F, Wu Z, et al. Tissue adhesives in gastrointestinal anastomosis: a systematic review. J Surg Res 2013;180:290300.

28. Vilallonga R, Himpens J, Bosch B, van de Vrande S, Bafort J. Role of percutaneous glue treatment after persisting leak after laparoscopic sleeve gastrectomy. Obes Surg 2016;26:1378-1383.

29. Bège T, Emungania 0, Vitton V, et al. An endoscopic strategy for management of anastomotic complications from bariatric surgery: a prospective study. Gastrointest Endosc 2011;73:238-244.

30. Parodi A, Repici A, Pedroni A, Blanchi S, Conio M. Endoscopic management of GI perforations with a new over-the-scope clip device (with videos). Gastrointest Endosc 2010;72:881-886.

31. Keren D, Eyal O, Sroka G, et al. Over-the-scope clip (OTSC) system for sleeve gastrectomy leaks. Obes Surg 2015;25:1358-1363.

32. Kumbhari V, Cai JX, Schweitzer MA. Endoscopic management of bariatric surgical complications. Curr Opin Gastroenterol 2015;31:359-367.

33. Papavramidis TS, Kotzampassi K, Kotidis E, Eleftheriadis EE, Papavramidis ST. Endoscopic fibrin sealing of gastrocutaneous fistulas after sleeve gastrectomy and biliopancreatic diversion with duodenal switch. J Gastroenterol Hepatol 2008;23:1802-1805.

34. Papavramidis ST, Eleftheriadis EE, Papavramidis TS, Kotzampassi KE, Gamvros OG. Endoscopic management of gastrocutaneous fistula after bariatric surgery by using a fibrin sealant. Gastrointest Endosc 2004;59:296-300.

35. Eisendrath P, Cremer M, Himpens J, Cadière GB, Le Moine 0, Devière J. Endotherapy including temporary stenting of fistulas of the upper gastrointestinal tract after laparoscopic bariatric surgery. Endoscopy 2007;39:625-630.

36. Bueter M, Thalheimer A, Wierlemann A, Fein M. Reoperations after gastric banding: replacement or alternative procedures? Surg Endosc 2009;23:334-340.

37. Noel P, Schneck AS, Nedelcu M, et al. Laparoscopic sleeve gastrectomy as a revisional procedure for failed gastric banding: lessons from 300 consecutive cases. Surg Obes Relat Dis 2014;10:11161122. 